\title{
In-Situ Characterization of Mechanical and Electrical Response of Individual BCN Nanotubes
}

\author{
Hessam Ghassemi $^{1 *}$, Chee H. Lee ${ }^{2}$, Yoke K. Yap ${ }^{2}$, Reza Shahbazian-Yassar ${ }^{1}$ \\ ${ }^{1}$ Department of Mechanical Engineering-Engineering Mechanics, Michigan Technological \\ University, 1400 Townsend Dr., Houghton, MI 49931 \\ ${ }^{2}$ Department of Physics, Michigan Technological University, 1400 Townsend Dr., Houghton, MI \\ 49931
}

We reported for the first time the buckling mechanism in multi-walled BNNTs upon severe mechanical deformation [1]. Individual BNNTs were deformed inside a transmission electron microscope (TEM) equipped with an in-situ atomic force microscopy holder, as shown in Fig. 1. The critical strain to form the first ripple was measured as $4.1 \%$ and the buckling process was reversible up to $26 \%$ strain. As opposed to carbon nanotubes, the BNNTs buckled into V-shaped ripples rather than smooth wavy shapes. The BNNTs showed a larger rippling wavelength compared to that of CNTs with the same number of walls. This difference was explained by the tendency of $\mathrm{BN}$ structures to reduce the number of thermodynamically unfavorable $\mathrm{B}-\mathrm{B}$ and $\mathrm{N}-$ $\mathrm{N}$ bonds at the sharp corners in the rippling regions. The BNNTs' structure also exhibited a higher fracture strain compared to their counterpart.

However, at large-angle cyclic bending fracture failure of individual BNNTs was observed [1]. Interestingly BNNTs failed in a fracture mode. Figure 2 shows the real-time process of the failure of an individual BNNT after numerous cycling bending. The $F-D$ curve corresponding to this fracture event illustrates the changes in the force applied to the nanotube (Figures 2a-e). As the TEM images show, the fracture initiates from the outermost shells (Figure 2c) that are under tensile stress and propagates (Figure 2e) toward the axis of nanotube, almost perpendicular to the applied force axis. Since the fracture processes initiated only two discrete drops of applied forces within this particular cycle of bending, apparently multiple $h-B N$ shells are broken simultaneously in two patches resembling a brittle type fracture.

We also showed that boron nitride nanotubes (BNNTs) can be ideal candidates for field emission applications as they demonstrate high emission current and current density, along with reliable mechanical stabilities. Compared to pure carbon nanotube, pure BNNTs start decomposing at relatively higher temperatures, $1000^{\circ} \mathrm{C}$. Field emission experiments were done inside a transmission electron microscope (TEM) using an in-situ electrical holder capable of applying bias voltage of up to $140 \mathrm{~V}$. Our results show that individual BNNTs can exhibit current densities above $100 \mathrm{~A} / \mathrm{cm}^{2}$, and emission currents up to $2 \mu \mathrm{A}$. Real-time captured images show the structural stability of individual BNNTs during and after running the field-emission experiments

\author{
References: \\ H. Ghassemi et al., Nanotechnology, 2011, 22, 115702 -115708 \\ H. Ghassemi et al., J. Appl. Phys. 2010, 108, pp. 024314-024317
}



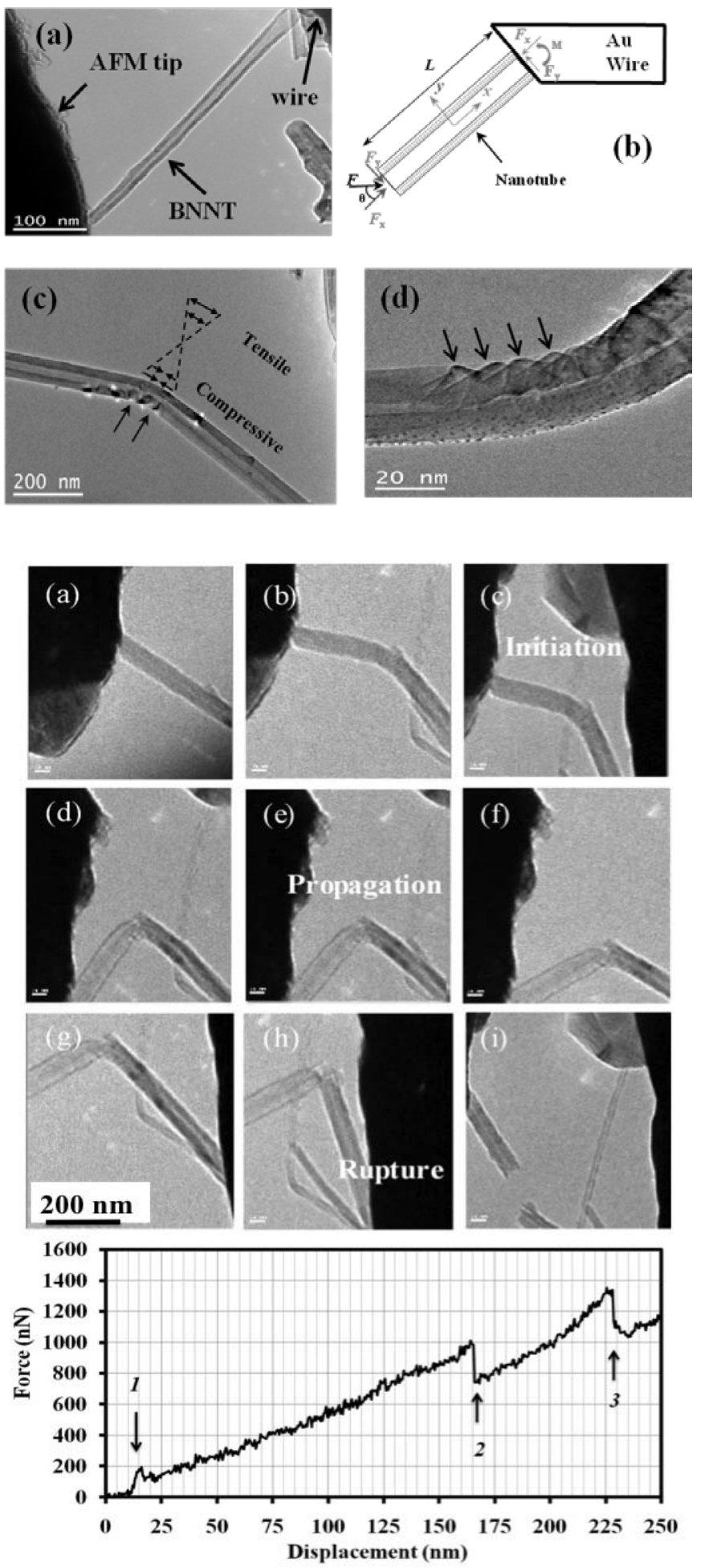

Figure 1 (a) Represents bending experiment of an individual multi-walled BNNT in contact with AFM tip (on left) and gold wire base (on right). (b) Free-body diagram of the bending experiment in (a) with analysis of associated forces. (c) The bent nanotube is shown and special pattern formation at the bent area is marked by arrows. (d) High Resolution (HR) TEM image of individual bent BNNT reveals that the special pattern consists of multiple rippling on the compressive side of nanotube.

Figure 2 Process of fracture in an individual BNNT after the large-angle $\left(\sim 120^{\circ}\right)$ bending cycles. (a) to (e) represent one cycle of bending. (f) to (i) showing nanotube breaking into two parts after pulling and stretching. (j) F-D plot represents the changes in force upon failure initiation and propagation during one cycle (a to e). Scale bars are $20 \mathrm{~nm}$. 